\title{
The Comparison of the Gluteus Maximus Activity during Various Prone Hip Extension in Subject with Chronic Low Back Pain
}

\author{
Yoo-Hun Kwon, PT • Heon-Seock Cynn, PT, $\mathrm{PhD}^{\dagger} \cdot$ Dong-Hwan Park, PT, MS • \\ A-Reum Shin, PT, MS • Seung-Min Baik PT $\cdot$ Ji-Hyun Lee, PT, $\mathrm{PhD}^{1}$ \\ Department of Physical Therapy, Graduate School, Yonsei University \\ ${ }^{1}$ Department of Physical Therapy, Baekseok University
}

Received: April 15, 2019 / Revised: April 15, 2019 / Accepted: June 3, 2019

(c) 2019 J Korean Soc Phys Med

\section{| Abstract |}

PURPOSE: Prone hip extension (PHE) has been used for assessment of lumbo-pelvic movement and strengthening exercise for weakness of the hip joint muscles in patients with chronic low back pain (CLBP). On the other hand, few studies have examined which are the best PHE exercises to activate the gluteus maximus (GM) selectively in physical therapy practice. To aim of this study compared the muscle activity of the GM, rectus femoris (RF), biceps femoris (BF), tibialis anterior (TA) during these four different prone hip extensions, PHE, PHE with quadriceps activation (PHEQA), PHE with ankle dorsiflexion (PHEAD), and PHE with ankle plantarflexion (PHEAP), in subjects with CLBP.

METHODS: Nineteen subjects with low back pain participated in this study. Subject performed four PHE exercises and surface electromyography (EMG) was used to evaluate the muscle activity. Data were analyzed by one-way

$\uparrow$ Corresponding Author : Heon-Seock Cynn cynn@yonsei.ac.kr, http://orcid.org/0000-0002-5810-2371

This is an Open Access article distributed under the terms of the Creative Commons Attribution Non-Commercial License (http://creativecommons.org/licenses/by-nc/3.0) which permits unrestricted non-commercial use, distribution, and reproduction in any medium, provided the original work is properly cited. repeated-measures analysis of variance $(\alpha=.05 / 3=.017)$ and $\mathrm{a}$ Bonferroni adjustment was performed if a significant difference was found.

RESULTS: The muscle activities recorded by EMG showed significant among the four exercises. The muscle activity of the GM increased significantly during PHEQA than during PHEAP $(\mathrm{P}=.012)$.

CONCLUSION: PHEQA is the most effective exercise for eliciting greater GM muscle activation among the four PHE exercises in subjects with CLBP.

Key Words: Prone hip extension, Low back pain, Gluteus maximus

\section{Introduction}

Low back pain (LBP) is among the most prevalent musculoskeletal disorders and most costly health problems $[1,2]$. In particular, chronic LBP (CLBP) is defined as the persistence of LBP beyond three months, which is associated with load transfer from the lumbopelvic area to the lower extremities $[3,4]$. Several studies have revealed a range of factors associated with CLBP. Moreover, attention has been drawn to the changes in motor control and muscle imbalance caused by the imposition over stress 
and compression on the vertebrae $[5,6]$. Previous studies have shown that the gluteus maximus (GM) can influence the sacroiliac joint stability significantly and its weakness was found to be associated with low back pain $[4,7,8]$.

The prone hip extension (PHE) not only activates the GM, but also stabilizes the lumbopelvic stability [9-12]. On the other hand, a previous study reported that increased anterior pelvic tilt was related to the delayed onset timing of the GM activity, and the erector spinae and hamstring muscles showed over-activity during PHE in people with CLBP [13-16]. Therefore, previous studies examined the effectiveness of various exercises to reduce the delay in GM firing relative to that of the biceps femoris (BF) [17-19].

The muscle activity of the lumbar, thoracic, and hip extensors (proximal muscles) can be increased by the overflow of neuronal energy from the lower extremity to upper extremity during contraction. The rectus femoris (RF) muscle improved due to hip and knee extension simultaneously [20]. Another study reported that when ankle movements were added to knee extension, the maximum isometric electromyography (EMG) levels of the quadriceps increased in healthy subjects [21]. In addition, on the leg press, the EMG activity of the RF was higher during ankle dorsiflexion by the tibialis anterior (TA) [21]. Therefore, the effect of a co-contraction of quadriceps activation (QA), ankle dorsiflexion (AD), ankle plantarflexion (AP) during PHE would activate the GM.

Although PHE improves the GM muscle activity in CLBP, no studies have recommended the most appropriate ankle position and QA during PHE. Therefore, this study compared the GM activity during four conditions of PHE, PHE with QA (PHEQA), PHE with ankle dorsiflexion (PHEAD), and PHE with ankle plantarflexion (PHEAP) in subject with CLBP. This study hypothesized that GM and RF would be greater during PHEQA, PHEAD, and PHEAP compared to PHE.

\section{Methods}

\section{Participants}

G-power software was used for power analyses (G-power software 3.1.2; Franz Faul, University of Kiel, Kiel, Germany). The necessary sample size of five subjects was calculated from the data obtained from a pilot study of 19 subjects to achieve a power of .80 and an effect size of .81 (calculated from the partial $\eta 2$ of 0.21 from the pilot study) with an a level of .05 . Therefore, 19 subjects participated in this study (age $=23.05$ years, height $=175.31$ $\mathrm{cm}$, weight $=73.82 \mathrm{~kg}$, and $\mathrm{BMI}=23.98$ ). The inclusion criteria for study participation were a history of nonspecific CLBP lasting for more than three months or the presence of recurrent LBP with at least three episodes, each lasting more than one week during the year before the study $[22,23]$. The exclusion criteria included (1) a history of lumbar, sacroiliac, or lower limb injury within the past year, (2) past or present cardiopulmonary diseases, (3) hip flexor shortness according to the Thomas test [24], (4) tensor fasciae latae shortness according to the Ober's test [25], and (5) adductor muscle shortness according to the Adduction Contracture test [25]. Those musculoskeletal examinations of the lower extremities were performed to avoid compensation related to muscle shortness. The level of pain while performing the activities of daily living before this study was measured using a visual analog scale (VAS) [26]. Table 1 lists the subjects' characteristics. Prior to participation, the subjects provided written informed consent after being given an explanation of the entire procedure by the principal investigator. The investigation was approved by Yonsei University Wonju Institutional Review Board (IRB: 1041849-201812-BM-117-02).

\section{Measurement methods and device}

1) Electromyography (EMG) recording and data processing

The surface EMG data were collected using a Noraxon 
Table 1. Subjects' Characteristics

\begin{tabular}{cc}
\hline Characteristics & Mean $\pm \mathrm{SD}^{\mathrm{a}}$ \\
\hline Age (year) & $23.053 \pm 2.068$ \\
Height $(\mathrm{cm})$ & $175.316 \pm 4.372$ \\
Weight $(\mathrm{kg})$ & $73.826 \pm 9.059$ \\
BMI $^{\mathrm{b}}\left(\mathrm{kg} / \mathrm{m}^{2}\right)$ & $23.989 \pm 2.447$ \\
$\mathrm{VAS}^{\mathrm{c}}$ & $4.158 \pm 1.425$ \\
\hline
\end{tabular}

a: mean \pm standard deviation,

b: body mass index.

: visual analog scale of pain

TeleMyo-DTS with a wireless telemetry system (Noraxon, Inc., Scottsdale, AZ, USA) and analyzed using the Noraxon MyoResearch 1.06 XP software. The EMG signals were amplified, band pass-filtered (10 and $450 \mathrm{~Hz}$ ), and notchfiltered $(60 \mathrm{~Hz}, 120 \mathrm{~Hz})$ before being recorded digitally at $1000 \mathrm{~Hz}$ and processed into the root-mean-square data. The data were collected from the GM, RF, BF, and TA on the painful side that usually feel more pain on the back. After shaving and rubbing the skin with alcohol, disposable $\mathrm{Ag} / \mathrm{AgCl}$ surface electrodes were placed on each muscle at standardized sites [27]. To minimize the cross-talk from the proximal deep or superficial muscles, the electrode, $1 \mathrm{~cm}$ in diameter, was used in this study. Two electrodes were placed approximately $2 \mathrm{~cm}$ apart in the direction of the muscle fibers. The GM electrodes were placed at half the distance between the greater trochanter and S2 vertebra in the middle of the muscle in an oblique angle. The RF electrodes were placed at $50 \%$ of the line from the anterior superior iliac spine to the superior part of the patella. The BF electrodes were placed at the center of the back of the thigh, approximately $50 \%$ of the distance from the gluteal fold to the back of the knee. The TA electrodes were placed at $25 \%$ on the line between the tip of the head of the fibula to the tip of the lateral malleolus. Correct electrode placement was confirmed by a visual inspection of the EMG signals on a computer screen during specific muscle testing.
The maximal voluntary isometric contractions (MVIC) were collected to normalize the EMG data from the GM, $\mathrm{RF}, \mathrm{BF}$, and TA using the manual muscle testing positions recommended by previous studies [28]. To determine the MVIC value for GM, each subject was placed in the prone position with their knee flexed at $90^{\circ}$ and a resistance placed on the distal region of the thigh with the pelvis stabilized. The RF was tested while the subjects were seated with their knee flexed at $90^{\circ}$ and a resistance placed on the distal tibia. The BF was tested while the hip joint was placed in the extension position, the knee was flexed to approximately $70^{\circ}$, and the resistance was applied to the distal aspect of the posterior portion of the shank during knee flexion. The TA was tested while subjects were sitting on the side of the bed with their hands resting on the bed on either side of the body for stability. The tibia was aligned with the vertical and ankle flexed at $0^{\circ}$. Each contraction was held for five seconds, with maximal effort against manual resistance, and a 2-minute rest was given between trials to minimize the muscle fatigue [29]. Verbal encouragement was given during all MVICs. The order of the MVICs was counterbalanced to avoid any potential neuromuscular fatigue.

\section{Procedures}

Before testing, the order of the conditions (PHE, PHEQA, PHEAD, and PHEAP) was determined using a randomization website (http://www.randomization.com) to avoid bias from the learning effects. All procedures for the EMG measurements were performed with the subjects in the prone position on the therapeutic table with a firm mattress. The subjects were asked to lie prone with their arms at their side and with a neutral position of the pelvis and hip joint. A goniometer was used to determine when the leg was extended at $10^{\circ}$ and a wooden target bar was placed at this level and feedback was provided. The feedback information at $10^{\circ}$ of hip extension also was given to the subjects by verbal instruction (Fig. 1). The part 

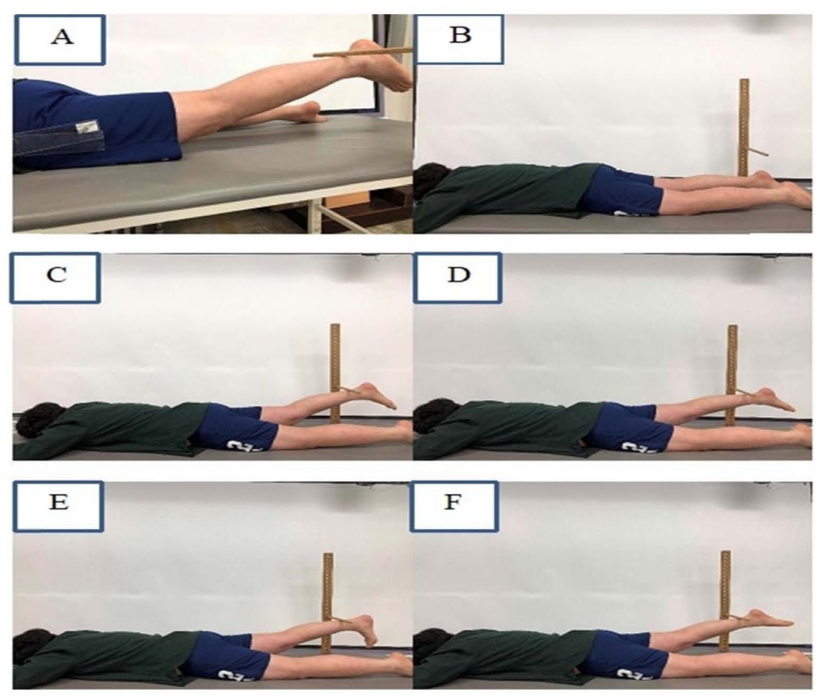

Fig 1. A: position as prone hip extension $10^{\circ}$, B: starting position, C: prone hip extension, D: prone hip extension with quadriceps activation, E: prone hip extension with ankle dorsiflexion, $\mathrm{F}$ : prone hip extension with ankle plantarflexion.

icipants were instructed to extend the leg of their painful side neutral to approximately $10^{\circ}$ while in contact with the Achilles tendon [30] for five seconds. The positions of the pelvis and limb were supervised visually during the PHE task to ensure that the subject maintained the neutral pelvis position, hip extension, and knee extension. The familiarization period concluded when the participant demonstrated an ability to conduct the four conditions protocol. The data were excluded if visible hip rotation movement or pelvic rotation was observed. A two minute rest period between each trial was allowed [31]. The mean of the two trials for each exercise was used for analysis.

\section{Experimental design}

\section{1) Prone hip extension}

All subjects were positioned prone on a therapeutic table with their feet shoulder width apart and arms at their sides. The head was allowed to extend slightly to normal breathing. For PHE exercise at the starting position, the subjects were asked to maintain knee extension and lift the leg of their painful side until the Achilles tendon touched the target bar as soon as they heard the verbal instruction "lift".

\section{2) Prone hip extension with quadriceps activation} (PHEQA)

PHEQA was performed in the same procedure as PHE, except that the subjects were straight knee order to perform the pre-activation of the quadriceps. The principal investigator (PI) explained to the subjects not to force the ankle and only strengthen on knee extension after hearing the verbal instruction "straighten your knee as strong as possible, and then lift". The PI confirmed to the subjects the RF and TA activity from the EMG monitor.

\section{3) Prone hip extension with ankle dorsiflexsion (PHEAD)}

The subjects performed in the same manner as for the PHE starting position, except that subjects were asked to undergo selective ankle dorsiflexion. The subjects' maintained the maximal ankle dorsiflexion during PHE 
Table 2. Electromyographic Activity of the Various Muscles

\begin{tabular}{cccccccc}
\hline & \multicolumn{9}{c}{ Exercise } & & F & p \\
\cline { 2 - 5 } & PHE & PHEQA & PHEAD & PHEAP & & & \\
\hline GM & $18.37 \pm 9.53$ & $21.58 \pm 13.76$ & $16.99 \pm 12.62$ & $16.65 \pm 11.19$ & 5.210 & $.003^{*}$ \\
RF & $8.10 \pm 5.71$ & $28.21 \pm 14.27$ & $14.90 \pm 7.78$ & $12.89 \pm 5.33$ & 16.228 & $.002^{*}$ \\
BF & $34.50 \pm 23.59$ & $38.64 \pm 30.22$ & $35.79 \pm 20.73$ & $33.30 \pm 18.56$ & .927 & .401 \\
TA & $1.19 \pm .70$ & $2.50 \pm 3.07$ & $33.52 \pm 14.70$ & $4.18 \pm 2.55$ & 30.833 & $.000^{*}$ \\
\hline
\end{tabular}

Values are presented as mean $\pm \mathrm{SD}$.

GM: gluteus maximus, RF: rectus femoris, BF: biceps femoris, TA: tibialis anterior.

PHE: Prone hip extension, PHEQA: Prone hip extension with quadriceps activation, PHEAD: Prone

Hip extension with ankle dorsiflexion, PHEAP: Prone hip extension with hip plantarflexion.

${ }^{*} \mathrm{p}<.017$, by one way repeated analyses of variance.

after hearing the verbal instruction "Pull your foot as firmly as possible, and then lift". The PI confirmed to the subjects the RF and TA activity from the EMG monitor.

\section{4) Prone hip extension with ankle plantarflexion (PHEAP)}

PHEAP was performed in the same way as PHE, except for the maximal ankle plantarflexion. The verbal instruction "Push your foot as firmly as possible, and then lift" was used.

\section{Statistical analysis}

The normality of the distribution was assessed using one-sample Kolmogorov-Smirnov tests. This study used a one-way repeated-measures analysis of variance to assess the statistical significance of the GM, RF, BF, and TA activities during the four different PHE exercises. The level of statistical significance was set to 0.05 . A Bonferroni correction was performed if a statistically significant difference was apparent (with $\alpha=.05 / 4=.012$ ). SPSS software (Chicago, IL, USA) ver. 20 was used to perform statistical analysis.

\section{Results}

The EMG activities of the GM, RF, and TA were significantly different among the four different PHE exercises $(\mathrm{F}=5.210, \mathrm{P}=.003 ; \mathrm{F}=16.228, \mathrm{P}=.002 ; \mathrm{F}=30.833$, $\mathrm{P}<.001)$ (Table 2). The activity of the GM was significantly greater during PHEQA (21.58 $\pm 13.76 \%$ MVIC) than during PHEAP (16.65 $\pm 11.19 \%$ MVIC, $\mathrm{P}=.005$ ) (Fig. 2). The activity of the RF was significantly greater during PHEQA (28.21 $\pm 14.27 \%$ MVIC) compared to that during PHE $(8.10 \pm 5.71 \%$ MVIC, $\mathrm{P}<.001)$, PHEAD $(14.90 \pm 7.78 \%$ MVIC, $\mathrm{P}<.001)$, and PHEAP (12.89 $\pm 5.33 \%$ MVIC, $\mathrm{P}=.001)$. The activity of the RF was significantly greater during PHEAD than during PHE $(\mathrm{P}=.039)$. The TA $(\mathrm{F}=30.833, \mathrm{P}<.001)$ activity was greatest during PHEAD

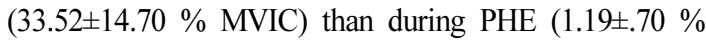
MVIC), PHEQA (2.50 $\pm 3.07 \%$ MVIC), and PHEAP (4.18 $\pm 2.55 \%$ MVIC). On the other hand, there was no significant difference in the EMG amplitude for the $\mathrm{BF}(\mathrm{F}=.927$, $\mathrm{P}=.401$ ) (PHE :34.50 $\pm 23.59 \%$ MVIC, PHEQA: 38.64 $30.22 \%$ MVIC, PHEAD: $35.79 \pm 20.73 \%$ MVIC, PHEAP $(33.30 \pm 18.56 \%$ MVIC).

\section{Discussion}

This study compared the GM activity during four different PHE exercises in subjects with CLBP. In this study, the activity of the GM was greater during PHEQA compared to PHE, PHEAD, and PHEAP. On the other 

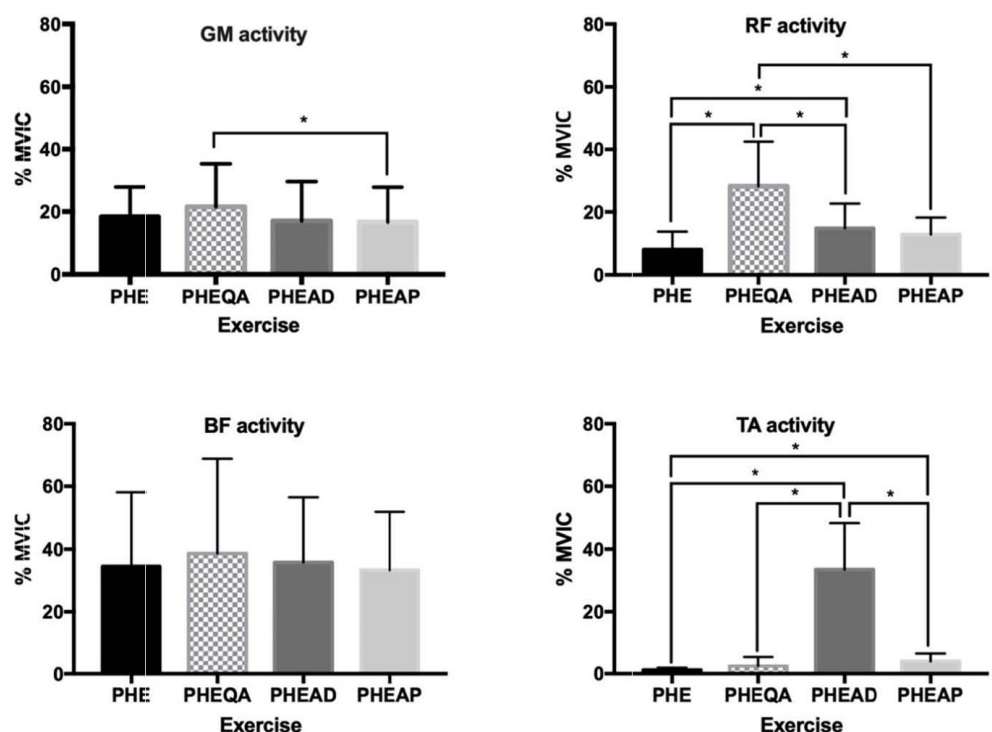

Fig. 2. Comparisons of the muscle activities during various prone hip extension. Prone hip extension (PHE), Prone hip extension with quadriceps activation (PHEQA), Prone hip extension with ankle dorsiflexion (PHEAD), Prone hip extension with hip plantarflexion (PHEAP).

(\% MVIC: maximal voluntary isometric contraction, GM: gluteus maximus, RF: rectus femoris, BF: biceps femoris, TA: tibialis anterior, error bar: standard deviation, ${ }^{*} \mathrm{P}<.012$ )

hand, no study has compared data from the GM, RF, BF, and TA muscle activities during different PHE in subjects with CLBP.

In the present study, the GM activity was significantly higher in PHEQA than in PHEAP (29.61\%). Based on the muscle attachment, GM showed increased leverage to its superior attachment to the dorsal ilium and was involved primarily in hip extension and lateral rotation [28]. A previous study finding suggested that the GM and $\mathrm{RF}$ perform a hip extensor muscle strategy synergistically during the ascending phase of standing squat exercise [32]. The gastrocnemius muscles are involved in plantarflexion of the ankle and originate at the medial and lateral femur condyles, insert on the calcaneus, and its proximal tendon covers the posterior side of the tibia. The triceps surae muscles also have been considered as their effect on the knee joint [33]. Therefore, the gastrocnemius muscles exert direct flexor activity on the knee joint [34]. On the other hand, when both plantarflexion and knee extension are added, the gastrocnemius muscles activity would be decreased which is an antagonist during knee extension. The mechanisms of reciprocal inhibition, decreased of antagonist activity is induced by the agonist activity through the neural pathways. Therefore, these results suggest that the PHEQA may be more effective than PHEAP for improving the GM activity in subjects with CLBP.

The RF activity was significantly greater in PHEQA than PHE (248.27\%), PHEAD (89.33\%), and PHEAP (118.85\%). Because this PHEQA exercise was focused on strengthening the RF, the activity of the RF is certainly increased. This expectation was based on the irradiation technique. Irradiation is a neurophysiological phenomenon that increases the spread and strength of the distal or central muscles through the stimulus (resistance) to the distal part and occurring on the temporal or spatial summation [35,36]. On the other hand, these findings did not support the research hypothesis. This PHEQA exercise, which affects the RF directly, would have had a greater impact than 
the motion of the ankle. Therefore, this indicates that the PHEQA may be the most effective method for improving the RF activity among four PHE exercises in subjects with CLBP.

The BF activity was not significantly different among the four PHE exercises. A previous study reported that the $\mathrm{BF}$ can increase the level of activation if the GM is weak during PHE [37]. Moreover, if the hamstrings are dominant, and the GM is inhibited, abnormal displacement of the greater trochanter can be palpated during PHE, which is a finding reported in cases of LBP [11]. Previous studies reported that fixed one joint muscle requires shortened muscle by the other joint during movement, the amount of muscle fibers used for movement is decreased, resulting in a decrease in muscle activity [38]. This further supports the importance of training the perform activities with hip and knee extensors. Synergistic muscles GM and BF affect each other and work together to perform the same movement [17].

The TA activity was significantly greater in PHEAD than in PHE (2716.8 \%), PHEQA (1240.8 \%), and PHEAP (701.91\%). Because this PHEAD exercise focused on strengthening the TA, the activity of the TA is increased. One possible mechanism can describe the cooperation between the high forces at the onset of a lengthening contraction and the loss of contraction. Consequently, the isometric pre-activation cause increased stiffness in the muscle [39-41].

Several limitations of this research should be noted. First, the subjects were young individuals, 20-26 years of age. Therefore, these findings cannot be generalized to patients of all age groups. A broader age range of subjects should be used in future studies to ensure generalizability. Second, this study used a cross-sectional method such that only the immediate effects of PHE exercises were determined. The effects after long-term application were not measured. More study will be needed to examine the long-term effects of PHE exercises in patients with CLBP. Third, surface
EMG was used to monitor the muscle activity, leaving the possibility of crosstalk from the adjacent muscles. Further research will be needed to assess the gender differences.

\section{Conclusion}

This study compared the effects of four different PHE exercises on $\mathrm{GM}, \mathrm{RF}, \mathrm{BF}$, and $\mathrm{TA}$ muscle activities in subjects with CLBP. These findings suggest that PHEQA may occur while enhancing GM activation in subjects with CLBP. Moreover, clinicians need to consider a hip extensor during PHE. These findings may help clinicians make more appropriate decisions when seeking to improve the performance of the hip extensors.

\section{References}

[1] Andersson G. Epidemiology of low back pain. 1998; 69(sup281):28-31.

[2] Hodges, Paul W, Carolyn A, Richardson, et al. Altered trunk muscle recruitment in people with low back pain with upper limb movement at different speeds. 1999;80(9):1005-12.

[3] OSullivan P. Diagnosis and classification of chronic low back pain disorders: Maladaptive movement and motor control impairments as underlying mechanism. Man Ther. 2005;10(4):242-55

[4] Vleeming A, Pool-Goudzwaard A, Stoeckart R, van Wingerden J, Snijders C, et al. The posterior layer of the thoracolumbar fascia. Its function in load transfer from spine to legs. Spine. 1995;20(7):753-8.

[5] Dankaerts W, O'sullivan P, Burnett A, et al. Altered patterns of superficial trunk muscle activation during sitting in nonspecific chronic low back pain patients: Importance of subclassification. 2006;31(17):2017-23.

[6] Hoy D, Brooks P, Blyth F, et al. The epidemiology of low back pain. Best Pract Res Clin Rheumatol. 2010; 
24(6):769-81.

[7] Mooney V. Coupled motion of contralateral latissimus dorsi and gluteus maximus: its role in sacroiliac stabilization. Movement, Stability, and Low Back Pain. 1997.

[8] Van Wingerden J, Vleeming A, Buyruk H, Raissadat $\mathrm{K}$, et al. Stabilization of the sacroiliac joint in vivo: verification of muscular contribution to force closure of the pelvis. Eur Spine J. 2004;13(3):199-205.

[9] Distefano L, Blackburn J, Marshall S, et al. Gluteal muscle activation during common therapeutic exercises. J Orthop Sports Phys Ther. 2009;39(7):532-40.

[10] Janda V. On the concept of postural muscles and posture in man. 1983;29(3):83-4.

[11] Sahrmann S. Diagnosis and treatment of movement impairment syndromes. Elsevier Health Sciences. 2002.

[12] Wilson J, Ferris E, Heckler A, et al. A structured review of the role of gluteus maximus in rehabilitation. 2005;33(3).

[13] Hodges P, Moseley G. Kinesiology. Pain and motor control of the lumbopelvic region: Effect and possible mechanisms. 2003;13(4):361-70.

[14] Hossain M, Nokes L. A model of dynamic sacro-iliac joint instability from malrecruitment of gluteus maximus and biceps femoris muscles resulting in low back pain. Med Hypotheses. 2005;65(2):278-81.

[15] Hungerford B, Gilleard W, Hodges P, et al. Evidence of altered lumbopelvic muscle recruitment in the presence of sacroiliac joint pain. 2003;28(14):1593-1600.

[16] Leinonen V, Kankaanpää M, Airaksinen O, et al. Back and hip extensor activities during trunk flexion/extension: Effects of low back pain and rehabilitation. 2000;81(1): 32-7.

[17] Chance-Larsen K, Littlewood C, Garth A, et al. Prone hip extension with lower abdominal hollowing improves the relative timing of gluteus maximus activation in relation to biceps femoris. Man Ther. 2010;15(1):61-5.

[18] Jeon IC, Hwang UJ, Jung SH, et al. Comparison of gluteus maximus and hamstring electromyographic activity and lumbopelvic motion during three different prone hip extension exercises in healthy volunteers. Phys Ther Sport. 2016;22:35-40.

[19] Kang SY, Jeon HS, Kwon O, et al. Activation of the gluteus maximus and hamstring muscles during prone hip extension with knee flexion in three hip abduction positions. Man Ther. 2013;18(4):303-7.

[20] Joshua. C, Stock MS, Hernandez JM, et al. Additional insight into biarticular muscle function: The influence of hip flexor fatigue on rectus femoris activity at the knee. J Electromyogr Kinesiol. 2018;42:36-43.

[21] Tassi N, Guazzelli JF, Gonçalves M, et al. Electromyographic evaluation of the rectus femoris muscle during exercises performed on the leg press. 1998;38(4):223-9.

[22] Arab AM, Haghighat A, Amiri Z, et al. Lumbar lordosis in prone position and prone hip extension test: Comparison between subjects with and without low back pain. Chiropr Man Therap. 2017;25(1):8

[23] Kahlaee AH, Ghamkhar L, Arab AM, et al. Effect of the abdominal hollowing and bracing maneuvers on activity pattern of the lumbopelvic muscles during prone hip extension in subjects with or without chronic low back pain: A preliminary study. J Manipulative Physiol Ther. 2017;40(2):106-17.

[24] Vogt L, Banzer. Dynamic testing of the motor stereotype in prone hip extension from neutral position. 1997; 12(2):122-7.

[25] Magee DJ. Orthopedic physical assessment-e-book. Elsevier Health Sciences, 2014.

[26] Edgar E, Ohnhaus, Adler R, et al. Methodological problems in the measurement of pain: A comparison between the verbal rating scale and the visual analogue scale. 1975; 1(4):379-84.

[27] Criswell E. Cram jr. Cram's introduction to surface electromyography. 2010.

[28] Kendall FP, McCreary EK, Provance PG, et al. Muscles: 
Testing and function, with posture and pain. Philadelphia: Lippincott Williams \& Wilkins. 2005.

[29] Emami M, Arab AM, Ghamkhar L, et al. The activity pattern of the lumbo-pelvic muscles during prone hip extension in athletes with and without hamstring strain injury. International journal of sports physical therapy. 2014;9(3):312.

[30] Arab AM, Ghamkhar L, Emami M, et al. Altered muscular activation during prone hip extension in women with and without low back pain. Chiropractic \& manual therapies. 2011;19(1):18.

[31] Sakamoto AC, Teixeira-Salmela LF, de Paula-Goulart FR, et al. Muscular activation patterns during active prone hip extension exercises. J Electromyogr Kinesiol. 2009; 19(1):105-12.

[32] Bryanton, Megan. Quadriceps effort during squat exercise depends on hip extensor muscle strategy. Sports biomechanics. 2015;14(1):122-38.

[33] Mokhtarzadeh, Hossein. Contributions of the Soleus and Gastrocnemius muscles to the anterior cruciate ligament loading during single-leg landing. Journal of biomechanics. 2013;46(11):1913-20.

[34] O' Connor, John J, Zavatsky, et al. Anterior cruciate ligament forces in activity. The Anterior Cruciate Ligament: Current and Future Concepts. Raven Press, New York. 1993.

[35] Hopf HC, Schlegel HJ, Lowitzsch K, et al. Irradiation of voluntary activity to the contralateral side in movements of normal subjects and patients with central motor disturbances. European neurology. 1974;12(3):142-7.

[36] Shimura K, Kasai T. Effects of proprioceptive neuromuscular facilitation on the initiation of voluntary movement and motor evoked potentials in upper limb muscles. Human movement science. 2002;21(1):101-13.

[37] Jonkers I, Stewart C, Spaepen A, et al. The complementary role of the plantarflexors, hamstrings and gluteus maximus in the control of stance limb stability during gait. Gait \& posture. 2003;17(3):264-72.

[38] Kennedy PM, Cresswell AG. The effect of muscle length on motor-unit recruitment during isometric plantar flexion in humans. Experimental brain research. 2001;137(1): 58-64.

[39] Ford LE, Huxley AF, Simmons RM, et al. The relation between stiffness and filament overlap in stimulated frogmuscle fibres. Journal of Physiology. 1981;311(1): 219-49.

[40] Huijing PA, Baan GC, et al. Myofascial force transmission causes interaction between adjacent muscles and connective tissue: effects of blunt dissection and compartmental fasciotomy on length force characteristics of rat extensor digitorum longus muscle. Archives of Physiology and Biochemistry. 2001;109(2):97-109.

[41] Benz RJ, Friden J, Lieber RL, et al. Simultaneous stiffness and force measurements reveal subtle injury to rabbit soleus muscles. Molecular and Cellular Biochemistry. 1998;179(1-2):147-58. 